\title{
Simulation and Analysis of An Ultra-Wideband Ranging System
}

\author{
Zifen Yang ${ }^{1, a}$, Lihua Han ${ }^{1, b}$, Guoqiang $\mathrm{Li}^{1,2, \mathrm{c}}$ and Chupeng Shi ${ }^{3, \mathrm{~d}}$ \\ 1School of Informatics, Linyi University, Linyi 276005, P.R. China \\ 2Provincial Key Laboratory for Network Based Intelligent Computing, University of \\ Jinan, 250022, P.R. China \\ 3Human Resources and Social Security Bureau of the cause of pension insurance \\ service center,Wenshui County, Shanxi Province,032100,P.R.China

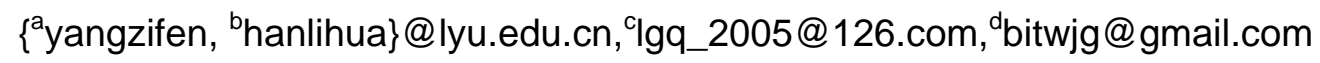 \\ Correspondence should be addressed to Guoqiang Li: lgq_2005@126.com
}

Keywords- Ultra-Wideband; Ranging System; Simulation

\begin{abstract}
By analyzing the principle of the UWB Ranging System, a mathematical model of the system is built, and then UWB signal models are chosen. By theoretical analysis, an important conclusion is drawn that signal processing of UWB ranging system is essentially time-domain sampling of echo signals. In the end, a simulation example is carried out in Matlab which certifies the conclusion. This result is very useful for UWB ranging system design and research.
\end{abstract}

\section{Introduction}

A signal can be classified as an UWB signal if the fractional bandwidth is greater than 0.25 . The fractional bandwidth can be determined as[1,2].

$\mu=\frac{2\left(f_{H}-f_{L}\right)}{f_{H}+f_{L}}$

where $f_{L}$ is the lower and $f_{H}$ is the higher -3dB point in a spectrum, respectively.

UWB ranging[3] is a newly emerging technology dedicated for precise distant measurement. Due to its properties of high-resolution, strong penetration, low probability of intercept and detection[2,4-13], UWB ranging systems have been increasingly widely used in military and civilian areas.

Based on the analysis of a high-precision UWB ranging system, the mathematical model of the system is developed. This research is the foundation of further study on the effect of echo signals on the system performance.

\section{Principle of the UWB ranging System}

The UWB ranging system is a kind of micro-power UWB radar, dedicated for objects ranging in proximity. Applications of UWB ranging systems include parking sensors, intruding detection systems and proximity fuses. By transmitting UWB impulse signals and receiving echo signals, UWB ranging systems can measure the speed and distance of objects precisely.

A typical UWB ranging system includes seven components [11]: random modulation circuit, pulse generating circuit, UWB pulse generating circuit, transmitting antenna, receiving antenna, signal processing circuit and range gate pulse generating circuit. The pulse generation circuit produces a periodic pulse signal. This periodic signal is transformed into random interval pulse train in random modulation circuit, which is named as driving signal. The driving signal outputs into UWB pulse generation circuit and range gate pulse generation circuit. The UWB pulse generation circuit produce UWB narrow pulse signal at the rising edge of the driving signal pulse. The UWB narrow pulse signal then will be transmitted by the UWB transmitting antenna. The range gate pulse 
generation circuit produces range gate pulse by applying a specified delay on the driving signal. The range gate pulses then output to the signal processing circuit. In the signal processing circuit, echo signals from receiving antenna are firstly processed using correlation integration technology with and the range gate pulses, and then by means of differentiation processing, the speed and distance of objects will be derived from the processed signal.

\section{Mathematical Model of UWB Ranging System}

Assume $p_{t}(t)$ is a single UWB narrow pulse transmitted at $t=0$, then the signal at distant $R$ from transmitting antenna can be presented by

$$
P_{R}(t)=\frac{1}{2 \sqrt{\pi} R} p_{t}\left(t-\frac{R}{c}\right) \sqrt{G_{t}(t, \theta, \varphi)}
$$

where $t$ is time, $c$ is the speed of light, $G_{t}(t, \theta, \varphi)$ [2]is the transmitting antenna gain at azimuth angle $\theta$ and pitch angle $\varphi$.

According to reference[2], the RCS(radar cross section) of objects is represented by $\sigma(\mathrm{t}, \theta, \varphi)$, because it is not only determined by incident angle, but also varing with time, for UWB narrow pulse signals. Despite of time compression and expansion effects on single UWB pulse by object speed, the reflected signal of object reaches the receiving antenna can be represented by

$$
p_{o}(t)=\frac{1}{2 \sqrt{\pi} R} P_{R}\left(t-\frac{R}{c}\right) \sqrt{G_{t}\left(t-\frac{R}{c}, \theta, \varphi\right)}
$$

The relationship between effective area of receiving antenna $\mathrm{A}_{r}(t, \theta, \varphi)$ and the antenna gain $\mathrm{G}_{r}(t, \theta, \varphi)$ is given by[2]

$$
A_{r}(t, \theta, \varphi)=\frac{\left(c t_{w}\right)^{2}}{\Delta \pi} G_{r}(t, \theta, \varphi)
$$

The signal received by receiving antenna is

$$
p_{r}(t)=\sqrt{A_{r}(t, \theta, \varphi)} p_{0}(t)
$$

Then the received signal can be derived from the transmitted signal by

$$
p_{r}(t)=\frac{c t_{w}}{(4 \pi)^{3} R^{2}} \sqrt{G_{r}(t, \theta, \varphi) G_{t}\left(t-\frac{2 R}{c}, \theta, \varphi\right) \sigma\left(t-\frac{2 R}{c}, \theta, \varphi\right)} p_{t}\left(t-\frac{2 R}{c}\right)=F\left\{p_{t}(t)\right\}
$$

Where $F$ represent the relationship between single transmitted UWB signal and reflected signal that received by receiving antenna.

From analysis of UWB ranging system, it is obvious that the system works in random modulation mode, which means that the pulse train transmitted within a given duration $T_{w}$ is composed of several randomly distributed narrow pulses in time-domain. Assume the random time sequence is $\left\{\tau_{i}\right\}, i=1,2,3 \ldots, \tau_{i+1}>\tau_{i}$, the pulse train output to transmitting antenna can be represented by

$$
f_{T}(t)=\sum_{i=1}^{N} p_{t}\left(t-\tau_{i}\right)
$$


The echo signal from receiving antenna to signal processing circuit can be represennted by

$$
f_{R}(t)=F\left[f_{T}(t)\right]=\sum_{i=1}^{N} F\left[p_{t}\left(t-\tau_{i}\right)\right]=\sum_{i=1}^{N} p_{r}\left(t-\tau_{i}\right)
$$

The echo signal in Eq.8 includes radiation characteristics of transmitting and receiving antennas, propagation delay, amplitude attenuation and the RCS of object. Refer Eq. 6 for more details.

As shown in Fig.1, in signal processing circuit, range gate pulse signal $p_{\text {gate }}(t)$ correlates with echo signal $f_{R}(t)$. The signal $p_{\text {gate }}(t)$ is a copy of transmitted signal $p_{t}(t)$ with delay $\Delta \mathrm{t}$, which is determined by range gate $R_{\text {gate }}$ in ${ }_{\Delta \mathrm{t}=}{ }^{2 R_{\text {gat }}} / \mathrm{c}$. The range gate signal $p_{\text {gate }}(t)$ can be represented by

$$
\mathrm{p}_{\text {gate }}\left(t-\tau_{i}\right)=p_{t}\left(t-\tau_{i}-\Delta t\right)
$$

The range gate signal train is

$$
\mathrm{f}_{\text {gate }}(t)=\sum_{i=1}^{N} p_{\text {gate }}\left(t-\tau_{i}\right)=\sum_{i=1}^{N} p_{t}\left(t-\tau_{i}-\Delta t\right)
$$

The output of $N$ pulses of $f_{R}(t)$ in duration $T_{w}$ can be represented by

$$
f_{\text {gate }}(t)=\sum_{i=1}^{N} p_{\text {gate }}\left(t-\tau_{i}\right)=\sum_{i=1}^{N} p_{t}\left(t-\tau_{i}-\Delta t\right)
$$

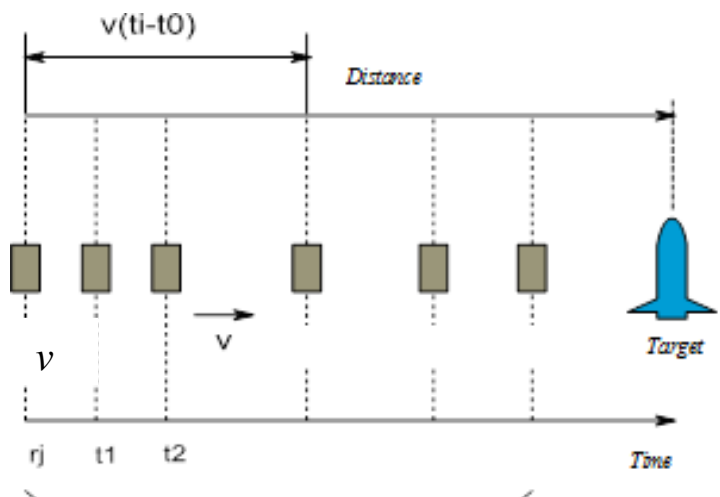

Fig.1. Antenna Positions at time $\tau_{\mathrm{i}}$ in a range cell

The speed of target is also important information implied in the echo signal. The following method is developed to model target speed.

Assume the radial velocity of the antenna and target is $v$, and the echo signal received by antenna at position $r_{j}$ corresponds to the transmitted pulse signal at time $\tau_{\mathrm{j}}$. Assumption is also made that during $T_{w}$, the shape of echo signals keeps invariant. If the received signal $\mathrm{p}_{r}^{\tau_{i}}(t)$ is appeared at time $\tau_{1}$, the echo signal of single transmitted pulse can be represented by

$$
p_{r}^{\tau_{j}}(t)=p_{r}^{\tau_{1}}\left(t+\frac{2 v\left(\tau_{j}-\tau_{1}\right)}{c}\right)
$$

With Eq.12, Eq.8 and Eq.11, the integration output signal can be derived as 


$$
f_{\text {int }}(t)=f_{\text {gate }}(t) f_{R}(t)=\sum_{i=1}^{N} \sum_{j=1}^{N} p_{t}\left(t-\tau_{i}-\Delta t\right) p_{r}^{\tau_{j}}(t)=\sum_{i=1}^{N} \sum_{j=1}^{N} p_{t}\left(t-\tau_{i}-\Delta t-\frac{2 v\left(\tau_{j}-\tau_{1}\right)}{c}\right) p_{r}^{t_{1}}(t)
$$

where $t \in\left[t_{0}, t_{0}+T_{w}\right]$.

\section{UWB Signal Model}

A class of waveforms is called Gaussian waveforms [2] because their mathematical definition is similar to the Gaussian function [3]. The zero-mean Gauss function is described by Eq. (2.14), where $\sigma$ is standard deviation. More waveforms can be created by a sort of high-pass filtering of this Gaussian pulse. Filtering acts in a manner similar to taking the derivative of Eq. (2.14). A Gaussian monocycle, the first derivative of a Gaussian pulse is described by Eq.(2.15). A Gaussian doublet is the second derivative of Eq. (2.14) and is defined by Eq. (2.16). The third to fifth derivative of Eq. (2.14) and are defined by Eq. (2.16)-Eq.(2.19).

$$
\begin{aligned}
& p(t)=\frac{A_{0}}{\sqrt{2 \pi} \sigma} \exp \left(-\frac{t^{2}}{2 \sigma^{2}}\right) \\
& p^{(1)}(t)=\frac{A_{1} t}{\sqrt{2 \pi} \sigma^{3}} \exp \left(-\frac{t^{2}}{2 \sigma^{2}}\right) \\
& p^{(2)}(t)=A_{2}\left(\frac{t^{2}}{\sqrt{2 \pi} \sigma^{5}}-\frac{1}{\sqrt{2 \pi} \sigma^{3}}\right) \exp \left(-\frac{t^{2}}{2 \sigma^{2}}\right) \\
& p^{(3)}(t)=A_{3}\left(\frac{t^{3}}{\sqrt{2 \pi} \sigma^{7}}-3 \frac{t}{\sqrt{2 \pi} \sigma^{5}}\right) \exp \left(-\frac{t^{2}}{2 \sigma^{2}}\right) \\
& p^{(4)}(t)=A_{4}\left(\frac{t^{4}}{\sqrt{2 \pi} \sigma^{9}}-6 \frac{t^{2}}{\sqrt{2 \pi} \sigma^{7}}+3 \frac{1}{\sqrt{2 \pi} \sigma^{5}}\right) \exp \left(-\frac{t^{2}}{2 \sigma^{2}}\right) \\
& p^{(5)}(t)=A_{5}\left(-\frac{t^{5}}{\sqrt{2 \pi} \sigma^{11}}+\frac{10 t^{3}}{\sqrt{2 \pi} \sigma^{9}}-\frac{15 t}{\sqrt{2 \pi} \sigma^{7}}\right) \exp \left(-\frac{t^{2}}{2 \sigma^{2}}\right)
\end{aligned}
$$

Where $\mathrm{t}$ is time, A1-A5 are amplitude constants, and $\sigma$ is standard deviation.

\section{Simulation Results}

A simulation example is designed to verify the conclusion drawn above. Let $N=100, v=100 \mathrm{~m} / \mathrm{s}$, and the center PRF (pulse repetition frequency) $=20 \mathrm{MHz}$, which means that the actual PRF randomly deviates from $10 \mathrm{MHz}$ to $30 \mathrm{MHz}$ and finally determines the random time sequence $\left\{\tau_{i}\right\}, i=1,2,3 \ldots, \tau_{i+1}>\tau_{i}$. The simulation is finished in Matlab. The simulation results are shown in Fig.2. In time dimension, the two simulated signals are both stretched by $1.5 \times 10^{6}$ times, compared to the original signals. When noise is introduced into simulation, the amplitudes decrease, but the time stretching factor remains the same. The simulation results coincide to the theoretical analysis in Section 5. 


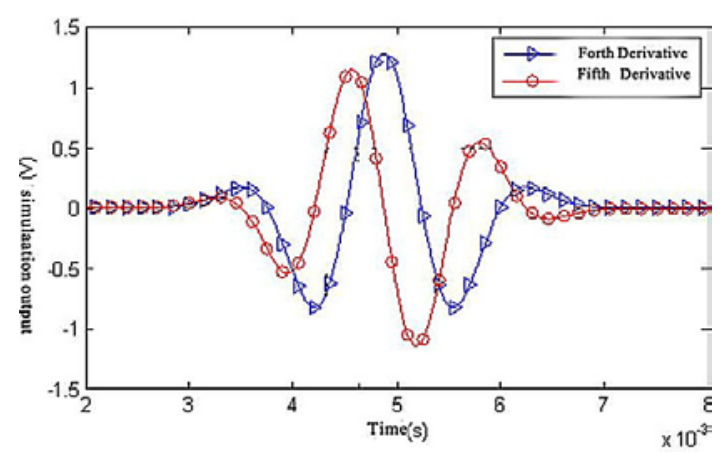

(a) No Noise

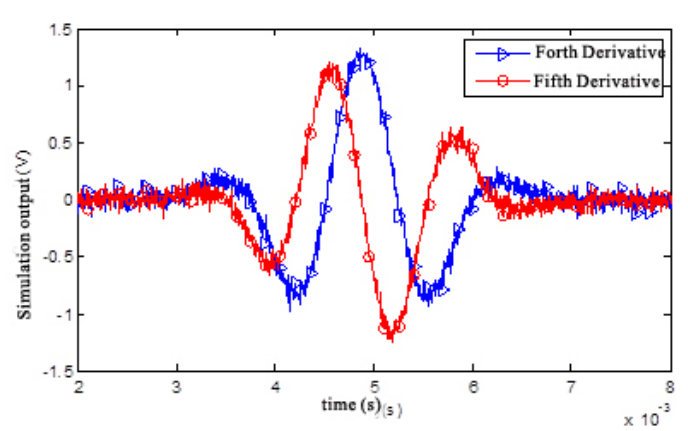

(b) Noise Power $=-45 \mathrm{~dB}$

Fig.2. Simulation Results

\section{Summary}

By analyzing the principle of the UWB Ranging System, a mathematical model of the system is built, and then UWB signal models are chosen. By theoretical analysis, an important conclusion is drawn that signal processing of UWB ranging system is essentially time-domain sampling of echo signals. In the end, a simulation example is carried out in Matlab which certifies the conclusion. This result is very useful for UWB ranging system design and research.

\section{Acknowledgments}

This work was supported by Shandong Provincial Natural Science Foundation, China (ZR2014FL013) and supported by $\mathrm{PhD}$ research foundation of Linyi University (LYDX2016BS077). The authors acknowledge the support of the Opening Fund of Shandong Provincial Key Laboratory for Network Based Intelligent Computing.

\section{References}

[1] Taylor J. D. Introduction to ultra wideband radar systems[M]. Boca Raton, FL USA: CRC Press,Inc., (1995).

[2] Taylor J. D. Ultra-wideband radar technology[M]. CRC Press LLC, (2001).

[3] Noel B. Ultra-wideband radar: Proceedings of the First Los Alamos symposium[M]. Boca Raton: CRC Press, (1991).

[4] Fontana R. J. Recent System Applications of Short-Pulse Ultra-Wideband (UWB) Technology[J]. IEEE TRANSACTIONS ON MICROWAVE THEORY AND TECHNIQUES, 52(9): 2087-2104,(2004)

[5] Wang Chunyang, Liu Gang, Shi Jianjun. A Kind of High Precision Fuse System [J]. SYSTEMS ENGINEERING AND ELECTRONICS, 26(3), (2001),p.14-15,

[6] Ghavami M., Michael L. B., Kohno R. Ultra Wideband Signals and Systems in Communication Engineering[M]. John Wiley \& Sons, Ltd, 2004.

[7] Oppermann I. Hamalainen M., Iinatti J. UWB Theory and Applications[M]. John Wiley \& Sons, Ltd, (2004).

[8] Lule E. K. Characterization of Ultra Wideband Antennas for Personal Communication Devices Using Finite Difference Time Domain Technique[D]. Miami, Florida: Florida International University, (2005).

[9] Jol H. M. Ground Penetrating Radar Theory and Applications[M]. Elsevier Science (2009).

[10] Daniels D. Ground Penetrating Radar[M]. 2nd edn: The Institution of Engineering and Technology, (2004).

[11] Gresham I. Jenkins A., Egrid R. Ultra-wideband radar sensors for short-range vehicular applications $\lceil\mathrm{J}]$. IEEE, Transactions on Microwave Theory and Techniques, 52(91),(2004),p.2105-2122.

[12] Fontana R. J., Foster L. A., Fair B., Wu D. Recent advances in Ultra Wideband radar and ranging systems. IEEE International Conference on Ultra-Wideband, ICUWB, (2007),p.19-25.

[13] Wenger J., Hahn S. Long range and ultra-wideband short range automotive radar. IEEE International Conference on Ultra-Wideband, ICUWB, (2007),p.518-522.

[14] M. Ghavami, L. B. Michael, and R. Kohno. Ultra Wideband Signals and Systems in Communication Engineering. John Wiley \& Sons, Ltd, (2004) 